\title{
THE COLOURED QUANTUM PLANE
}

\author{
DEEPAK PARASHAR
}

\begin{abstract}
We study the quantum plane associated to the coloured quantum group $G L_{q}^{\lambda, \mu}(2)$ and solve the problem of constructing the corresponding differential geometric structure. This is achieved within the $R$-matrix framework generalising the Wess-Zumino formalism and leads to the concept of coloured quantum space. Both, the coloured Manin plane as well as the bicovariant differential calculus exhibit the colour exchange symmetry. The coloured $h$-plane corresponding to the coloured Jordanian quantum group $G L_{h}^{\lambda, \mu}(2)$ is also obtained by contraction of the coloured $q$-plane.

[Jour. Geom. Phys., to appear.]

MSC (2000): 81R50; 17B37
\end{abstract}

Keywords: Quantum groups; Manin plane; Differential calculus

\section{INTRODUCTION}

Manin's approach [1] to quantum groups is based on the fact that these structures can be considered as comodule algebras for the so-called quantum or Manin plane. In analogy with the classical case, a quantum group also acts upon a formal vector space called the quantum vector space, or simply quantum space. For the simplest example of the two-dimensional case, this is generated by elements $x$ and $y$ satisfying the nontrivial commutation relation

$$
x y=q y x
$$

where $q \neq 1$ is the quantum deformation parameter and $\{x, y\}$ commute with the generating elements of the quantum group $G L_{q}(2)$. In the past decade or so, the study of quantum planes and quantum spaces has been at the forefront of mathematical physics research. One of the most interesting aspects is the formulation of a consistent differential calculus on the quantum plane, a powerful procedure given by Wess and Zumino [2]. An immediate consequence is the $q$-deformation of the quantum mechanical phase space, also given in [2]. Since then, extensive effort has 
been directed towards investigating the algebraic and differential geometric structure of quantum planes and quantum spaces in the noncommutative setting such as development of noncommutative gauge theories and quantum field theories. The study of such geometric structures also lies at the interface of Connes' noncommutative differential geometry and the theory of quantum groups. The Wess-Zumino formalism admits interesting multiparametric generalisations [3] and the technique has also been successfully applied to the $h$-planes [4, 5, 6] associated with the nonstandard $h$-deformations.

More recently, 'coloured' quantum groups have been studied [17, 8, 9, 10, 11] in the context of generalisation of the basic theory of quantum groups by parametrising the corresponding generators by some continuously varying colour parameters. Emerged originally as non-additive solutions of the spectral parameter dependent quantum Yang-Baxter equation in the study of integrable systems, coloured quantum groups are infinite-dimensional algebras. This is an interesting feature since on the one hand these are examples of infinite-dimensional Hopf algebras, now known as coloured Hopf algebras, while on the other hand they can be couched in the language familiar to those for the finite-dimensional ones. These are also related to invariants of knots and braid group respresentations similar to the usual uncoloured quantum groups. For further motivation see [8, 11] and references therein.

Despite the interest generated in the study of the coloured extension of quantum groups, little is known about the quantum planes or quantum spaces associated to these structures and the whole theory remains pretty much in its embryonic stage. In a recent work [12], the coloured quantum group $G L_{q}^{\lambda, \mu}(2)$ was investigated to establish a duality between the coloured algebra of quantised functions on the group and that of its universal enveloping algebra, i.e., its dual. In [12], the author also gave a coloured generalisation of the $R$-matrix approach to construct a bicovariant differential calculus on $G L_{q}^{\lambda, \mu}(2)$. A natural question that arises at this stage pertains to the quantum spaces upon which coloured quantum groups act. It is the purpose of this paper, therefore, to present a differential calculus on the coloured quantum planes associated to $G L_{q}^{\lambda, \mu}(2)$. This is carried out by generalising the Wess-Zumino formalism and leads to the concept of a coloured quantum space. 


\section{Coloured FRT Algebra}

It is well-established [7] that $G L_{q}^{\lambda, \mu}(2)$ provides a coloured generalisation of $G L_{q}(2)$ resulting in a coloured Faddeev-Reshetikhin-Takhtajan (FRT) 13 algebra. As already mentioned, the corresponding generators are parametrised by continuously varying colour parameters $\lambda$ and $\mu$, and as such the whole algebra becomes infinitedimensional. Recall that the $G L_{q}^{\lambda, \mu}(2)$ commutation algebra is given as

$$
\begin{aligned}
a_{\lambda} b_{\mu}=q^{1+2 \lambda} b_{\mu} a_{\lambda} & a_{\lambda} c_{\mu}=q^{1-2 \lambda} c_{\mu} a_{\lambda} \\
b_{\lambda} c_{\mu}=q^{-2(\lambda+\mu)} c_{\mu} b_{\lambda} & b_{\lambda} d_{\mu}=q^{1-2 \mu} d_{\mu} b_{\lambda} \\
d_{\lambda} c_{\mu}=q^{-1-2 \lambda} c_{\mu} d_{\lambda} & {\left[a_{\lambda}, d_{\mu}\right]=\left(q-q^{-1}\right) q^{\lambda+\mu} b_{\mu} c_{\lambda} } \\
a_{\lambda} b_{\mu}=q^{\lambda-\mu} a_{\mu} b_{\lambda} & a_{\lambda} c_{\mu}=q^{\mu-\lambda} a_{\mu} c_{\lambda} \\
b_{\lambda} c_{\mu}=b_{\mu} c_{\lambda} & b_{\lambda} d_{\mu}=q^{\lambda-\mu} b_{\mu} d_{\lambda} \\
c_{\lambda} d_{\mu}=q^{\mu-\lambda} c_{\mu} d_{\lambda} & a_{\lambda} d_{\mu}=a_{\mu} d_{\lambda} \\
a_{\lambda} a_{\mu}= & a_{\mu} a_{\lambda} \\
b_{\lambda} b_{\mu}= & q^{2(\lambda-\mu)} b_{\mu} b_{\lambda} \\
c_{\lambda} c_{\mu}= & q^{2(\mu-\lambda)} c_{\mu} c_{\lambda} \\
d_{\lambda} d_{\mu}= & d_{\mu} d_{\lambda}
\end{aligned}
$$

which is obtained from the coloured $R T T$ relations where

$$
R(\lambda, \mu)=\left(\begin{array}{cccc}
q^{1-\lambda+\mu} & 0 & 0 & 0 \\
0 & q^{\lambda+\mu} & 0 & 0 \\
0 & q-q^{-1} & q^{-\lambda-\mu} & 0 \\
0 & 0 & 0 & q^{1+\lambda-\mu}
\end{array}\right)
$$

is non-additive and satisfies the coloured quantum Yang-Baxter equation, and $T_{\lambda}=$ $\left(\begin{array}{ll}a_{\lambda} & b_{\lambda} \\ c_{\lambda} & d_{\lambda}\end{array}\right)$ and $T_{\mu}=\left(\begin{array}{ll}a_{\mu} & b_{\mu} \\ c_{\mu} & d_{\mu}\end{array}\right)$ are quantum matrices of the generators. The coalgebra structure is given by $\Delta\left(T_{\lambda}\right)=T_{\lambda} \dot{\otimes} T_{\lambda}, \varepsilon\left(T_{\lambda}\right)=1$. The quantum determinant is $D_{\lambda}=a_{\lambda} d_{\lambda}-q^{1-2 \lambda} c_{\lambda} b_{\lambda}$ and the antipode is

$$
S\left(T_{\lambda}\right)=D_{\lambda}^{-1}\left(\begin{array}{cc}
d_{\lambda} & -q^{-1+2 \lambda} b_{\lambda} \\
-q^{1-2 \lambda} c_{\lambda} & a_{\lambda}
\end{array}\right)
$$

Clearly, the colourless $G L_{q}(2) F R T$ algebra is retrieved with vanishing of the colour parameters. 


\section{Coloured quantum plane}

In analogy with the standard uncoloured quantum group $G L_{q}(2)$, its coloured version $G L_{q}^{\lambda, \mu}(2)$ can be considered as an endomorphism of a pair of quadratic algebras $\mathcal{A}_{q}^{2 \mid 0}$ and $\mathcal{A}_{q}^{0 \mid 2}$ (its dual) in the sense of Manin [1]. The algebra $\mathcal{A}_{q}^{2 \mid 0}$ is generated by elements $\left\{x_{\lambda}, x_{\mu}, y_{\lambda}, y_{\mu}\right\}$, the 'coloured' coordinates, such that

$$
\begin{aligned}
x_{\lambda} x_{\mu} & =q^{\lambda-\mu} x_{\mu} x_{\lambda} \\
x_{\lambda} y_{\mu} & =q^{1-\lambda-\mu} y_{\mu} x_{\lambda} \\
x_{\lambda} y_{\mu} & =x_{\mu} y_{\lambda} \\
y_{\lambda} y_{\mu} & =q^{\mu-\lambda} y_{\mu} y_{\lambda}
\end{aligned}
$$

This is the so-called coloured quantum plane suggested in [7] and defines a left coaction

$$
\Delta_{L}: \mathcal{A}_{q}^{2 \mid 0} \longrightarrow G L_{q}^{\lambda, \mu}(2) \otimes \mathcal{A}_{q}^{2 \mid 0}
$$

i.e.

$$
\begin{aligned}
& \Delta_{L}\left(\begin{array}{l}
x_{\lambda} \\
y_{\lambda}
\end{array}\right) \longrightarrow\left(\begin{array}{ll}
a_{\lambda} & b_{\lambda} \\
c_{\lambda} & d_{\lambda}
\end{array}\right) \otimes\left(\begin{array}{l}
x_{\lambda} \\
y_{\lambda}
\end{array}\right) \\
& \Delta_{L}\left(\begin{array}{l}
x_{\mu} \\
y_{\mu}
\end{array}\right) \longrightarrow\left(\begin{array}{ll}
a_{\mu} & b_{\mu} \\
c_{\mu} & d_{\mu}
\end{array}\right) \otimes\left(\begin{array}{l}
x_{\mu} \\
y_{\mu}
\end{array}\right)
\end{aligned}
$$

where the coloured quantum matrices $T_{\lambda}$ and $T_{\mu}$ commute with the coloured coordinates $\left\{x_{\lambda}, x_{\mu}, y_{\lambda}, y_{\mu}\right\}$. In general, this can be written as

$$
x_{\lambda}^{i} x_{\mu}^{j}=B_{k l}^{i j} x_{\mu}^{k} x_{\lambda}^{l}
$$

where

$$
B_{k l}^{i j}= \begin{cases}B(\lambda, \mu)=\frac{1}{q} \hat{R}(\lambda, \mu) ; & i<j \\ B(\mu, \lambda)=\frac{1}{q} \hat{R}(\mu, \lambda) ; & i=j\end{cases}
$$

and

$$
\hat{R}(\lambda, \mu)=\left(\begin{array}{cccc}
q^{1-\lambda+\mu} & 0 & 0 & 0 \\
0 & q-q^{-1} & q^{-\lambda-\mu} & 0 \\
0 & q^{\lambda+\mu} & 0 & 0 \\
0 & 0 & 0 & q^{1+\lambda-\mu}
\end{array}\right)
$$


is the coloured braided $R$-matrix which solves the coloured braided Yang-Baxter equation. The right coaction is given by $\mathcal{A}_{q}^{0 \mid 2}$, the algebra dual to $\mathcal{A}_{q}^{2 \mid 0}$

$$
\Delta_{R}: \mathcal{A}_{q}^{0 \mid 2} \longrightarrow \mathcal{A}_{q}^{0 \mid 2} \otimes G L_{q}^{\lambda, \mu}(2)
$$

i.e.

$$
\begin{aligned}
& \Delta_{R}\left(\begin{array}{ll}
\xi_{\lambda} & \eta_{\lambda}
\end{array}\right) \quad \longrightarrow \quad\left(\begin{array}{ll}
\xi_{\lambda} & \eta_{\lambda}
\end{array}\right) \otimes\left(\begin{array}{ll}
a_{\lambda} & b_{\lambda} \\
c_{\lambda} & d_{\lambda}
\end{array}\right) \\
& \Delta_{R}\left(\begin{array}{ll}
\xi_{\mu} & \eta_{\mu}
\end{array}\right) \longrightarrow\left(\begin{array}{ll}
\xi_{\mu} & \eta_{\mu}
\end{array}\right) \otimes\left(\begin{array}{ll}
a_{\mu} & b_{\mu} \\
c_{\mu} & d_{\mu}
\end{array}\right)
\end{aligned}
$$

The algebra $\mathcal{A}_{q}^{0 \mid 2}$ is generated by the coloured Grassmann variables $\left\{\xi_{\lambda}, \xi_{\mu}, \eta_{\lambda}, \eta_{\mu}\right\}$ satisfying

$$
\begin{aligned}
\xi_{\lambda} \xi_{\mu} & =\eta_{\lambda} \eta_{\mu}=0 \\
\xi_{\lambda} \eta_{\mu} & =-q^{-(1+\lambda+\mu)} \eta_{\mu} \xi_{\lambda} \\
\xi_{\lambda} \eta_{\mu} & =\xi_{\mu} \eta_{\lambda}
\end{aligned}
$$

and defines the coloured quantum hyperplane. This can also be expressed in a more general form

$$
\xi_{\lambda}^{i} \xi_{\mu}^{j}=-C_{k l}^{i j} \xi_{\mu}^{k} \xi_{\lambda}^{l}
$$

where

$$
C_{k l}^{i j}= \begin{cases}C(\lambda, \mu)=q \hat{R}(\lambda, \mu) ; & i<j \\ C(\mu, \lambda)=q \hat{R}(\mu, \lambda) ; & i=j\end{cases}
$$

Both sets of relations (㺼) as well as (15) of the coloured quantum plane satisfy the $\lambda \leftrightarrow \mu$ exchange symmetry and are invariant under the coaction of $G L_{q}^{\lambda, \mu}(2)$. In other words, the coloured quantum group $G L_{q}^{\lambda, \mu}(2)$ acts on a quantum vector space generated by the coloured variables $\left\{x_{\lambda}, x_{\mu}, y_{\lambda}, y_{\mu}\right\}$ and this defines a 'coloured quantum space'. In the limit of the vanishing colour parameters, we recover exactly the uncoloured two-dimensional quantum plane (11) corresponding to $G L_{q}(2)$.

\section{Differential Calculus}

Before we proceed to the construction of a differential calculus on the coloured quantum plane, it is important to stress that equations (10) and (16) are coloured generalisations of the usual relations for the Manin plane and its exterior algebra, and so are the matrices $B_{k l}^{i j}$ and $C_{k l}^{i j}$. Therefore, the Wess-Zumino calculus [2] has 
to be modified in order to incorporate colour dependence. The resulting calculus is much more complicated, has colour copies of the various mathematical quantities and for convenience we follow the same notation as [2]. For the derivatives, we define

$$
\begin{aligned}
\partial_{x_{\lambda}}=\frac{\partial}{\partial_{x_{\lambda}}}, & \partial_{x_{\mu}}=\frac{\partial}{\partial_{x_{\mu}}} \\
\partial_{y_{\lambda}}=\frac{\partial}{\partial_{y_{\lambda}}}, & \partial_{y_{\mu}}=\frac{\partial}{\partial_{y_{\mu}}}
\end{aligned}
$$

and for the differentials

$$
\begin{array}{ll}
\xi_{\lambda}=\mathrm{d} x_{\lambda}, & \xi_{\mu}=\mathrm{d} x_{\mu} \\
\eta_{\lambda}=\mathrm{d} y_{\lambda}, & \eta_{\mu}=\mathrm{d} y_{\mu}
\end{array}
$$

The differential calculus on the coloured quantum plane $\mathcal{A}_{q}^{2 \mid 0}$ is given by the commutation relations between the variables and the derivatives

$$
\partial_{j_{\lambda}} x_{\mu}^{i}=\delta_{j}^{i}+C_{j l}^{i k} x_{\mu}^{l} \partial_{k_{\lambda}}
$$

with matrix $C$ defined in (17). For $i, j=1 . .2$, this yields for $\mathcal{A}_{q}^{2 \mid 0}$

$$
\begin{aligned}
& \partial_{x_{\lambda}} y_{\mu}=q^{1+\lambda+\mu} y_{\mu} \partial_{x_{\lambda}} \\
& \partial_{y_{\lambda}} x_{\mu}=q^{1-\lambda-\mu} x_{\mu} \partial_{y_{\lambda}} \\
& \partial_{y_{\lambda}} y_{\mu}=1+q^{2-\lambda+\mu} y_{\mu} \partial_{y_{\lambda}} \\
& \partial_{x_{\lambda}} x_{\mu}=1+q^{2+\lambda-\mu} x_{\mu} \partial_{x_{\lambda}}+\left(q^{2}-1\right) y_{\mu} \partial_{y_{\lambda}}
\end{aligned}
$$

In addition, the derivatives also satisfy relations among themselves

$$
\partial_{i_{\lambda}} \partial_{j_{\mu}}=F_{j i}^{l k} \partial_{k_{\mu}} \partial_{l_{\lambda}} ; \quad i \leq j
$$

where

$$
F_{j i}^{l k}=F(\lambda, \mu)=\frac{1}{q} \hat{R}(\lambda, \mu)
$$

This gives

$$
\partial_{x_{\lambda}} \partial_{y_{\mu}}=q^{-(1+\lambda+\mu)} \partial_{y_{\mu}} \partial_{x_{\lambda}}
$$

The differential calculus on the coloured quantum hyperplane $\mathcal{A}_{q}^{0 \mid 2}$ is given by the commutation relations between the variables and the differentials

$$
x_{\lambda}^{i} \xi_{\mu}^{j}=C_{k l}^{i j} \xi_{\mu}^{k} x_{\lambda}^{l}
$$


Again, the matrix $C$ has the same form as (17). Explicitly, for $\mathcal{A}_{q}^{0 \mid 2}$ we obtain

$$
\begin{aligned}
x_{\lambda} \xi_{\mu} & =q^{2+\lambda-\mu} \xi_{\mu} x_{\lambda} \\
y_{\lambda} \xi_{\mu} & =q^{1+\lambda+\mu} \xi_{\mu} y_{\lambda} \\
y_{\lambda} \eta_{\mu} & =q^{2-\lambda+\mu} \eta_{\mu} y_{\lambda} \\
x_{\lambda} \eta_{\mu} & =q^{1-\lambda-\mu} \eta_{\mu} x_{\lambda}+\left(q^{2}-1\right) \xi_{\mu} y_{\lambda}
\end{aligned}
$$

Furthermore, the commutation relations between the derivatives and the differentials are

$$
\partial_{j_{\lambda}} \xi_{\mu}^{i}=D_{j l}^{i k} \xi_{\mu}^{l} \partial_{k_{\lambda}}
$$

where

$$
D_{j l}^{i k}= \begin{cases}D(\lambda, \mu)=C^{-1}(\lambda, \mu) ; & i<j \\ D(\mu, \lambda)=C^{-1}(\mu, \lambda) ; & i=j\end{cases}
$$

So, we have for $\mathcal{A}_{q}^{0 \mid 2}$

$$
\begin{aligned}
\partial_{x_{\lambda}} \xi_{\mu} & =\frac{1}{q^{2+\lambda-\mu}} \xi_{\mu} \partial_{x_{\lambda}} \\
\partial_{x_{\lambda}} \eta_{\mu} & =\frac{1}{q^{1-\lambda-\mu}} \eta_{\mu} \partial_{x_{\lambda}} \\
\partial_{y_{\lambda}} \xi_{\mu} & =\frac{1}{q^{1+\lambda+\mu}} \xi_{\mu} \partial_{y_{\lambda}} \\
\partial_{y_{\lambda}} \eta_{\mu} & =\frac{1}{q^{2-\lambda+\mu}} \eta_{\mu} \partial_{y_{\lambda}}+\left(\frac{1}{q^{2}}-1\right) \xi_{\mu} \partial_{x_{\lambda}}
\end{aligned}
$$

Finally, the exterior differential is defined as

$$
\begin{gathered}
\mathbf{d}=\xi_{\lambda}^{i} \partial_{i_{\lambda}}=\xi_{\mu}^{i} \partial_{i_{\mu}} \\
\mathbf{d}=\xi_{\lambda} \partial_{x_{\lambda}}+\eta_{\lambda} \partial_{y_{\lambda}}=\xi_{\mu} \partial_{x_{\mu}}+\eta_{\mu} \partial_{y_{\mu}}
\end{gathered}
$$

It can be checked that the exterior differential is nilpotent $\left(\mathbf{d}^{2}=0\right)$ and satisfies the Leibniz rule $\mathbf{d}(f g)=(\mathbf{d} f) g+f(\mathbf{d} g)$. The full differential calculus on the coloured quantum plane and the hyperplane respects the $\lambda \leftrightarrow \mu$ exchange symmetry, satisfies all consistency conditions and is invariant under the coaction of the coloured quantum group $G L_{q}^{\lambda, \mu}(2)$. Note that for vanishing colour parameters, the calculus reduces exactly to the calculus on the standard uncoloured two-dimensional quantum plane corresponding to $G L_{q}(2)$. It is also important to note that while we have treated the coloured version of the two-dimensional quantum plane, the result is 
actually infinite-dimensional since $\lambda$ and $\mu$ are continuously varying colour parameters. As such, not only are the coloured quantum planes infinite-dimensional but the differential calculus obtained here is also infinite-dimensional.

\section{Coloured $h$-Plane}

We now look at the nonstandard (or Jordanian) counterpart of the $q$-plane, known as the $h$-plane $[4,5]$. Motivated by the observation that coloured Jordanian quantum groups can be obtained from their $q$-deformed counterparts «, 10, 14] using the wellknown contraction procedure, we perform the contraction on the coloured $q$-plane discussed above. We consider the transformation on the coloured coordinates

$$
\left(\begin{array}{l}
x_{\lambda} \\
y_{\lambda}
\end{array}\right) \longrightarrow g\left(\begin{array}{l}
x_{\lambda} \\
y_{\lambda}
\end{array}\right) \quad \text { and } \quad\left(\begin{array}{l}
x_{\mu} \\
y_{\mu}
\end{array}\right) \longrightarrow g\left(\begin{array}{l}
x_{\mu} \\
y_{\mu}
\end{array}\right)
$$

where $g$ is the two-dimensional transformation matrix

$$
g=\left(\begin{array}{ll}
1 & \alpha \\
0 & 1
\end{array}\right)
$$

and $\alpha=\frac{h}{q-1}$ depends on both ' $q$ ' and the ' $h$ ' deformation parameters. Applying this transformation to the coloured $q$-plane (7), we obtain in the singular limit $q \rightarrow 1$

$$
\left[x_{\lambda}, y_{\mu}\right]=h(1-2 \mu) y_{\lambda} y_{\mu}
$$

i.e. the coloured $h$-plane corresponding to the coloured Jordanian quantum group $G L_{h}^{\lambda, \mu}(2)$ [9, 10]. Again, for vanishing colour parameters, this reduces to the wellknown $h$-plane $[x, y]=h y^{2}$ for the Jordanian quantum group $G L_{h}(2)$. While performing the above transformation, we also obtain a 'hybrid' standard-nonstandard quantum plane

$$
x_{\lambda} y_{\mu}-q^{1-\lambda-\mu} y_{\mu} x_{\lambda}=h[1-2 \mu]_{q} y_{\lambda} y_{\mu}=h q^{\mu-\lambda}[1-2 \mu]_{q} y_{\mu} y_{\lambda}
$$

which we call the 'coloured $(q, h)$-plane', where $[x]_{q}=\frac{1-q^{x}}{1-q}$ is the basic number from $q$-analysis.

\section{Conclusions}

We have presented the coloured quantum plane and the hyperplane covariant under

the action of the coloured quantum group $G L_{q}^{\lambda, \mu}(2)$. Generalising the Wess-Zumino formalism, we have also constructed a consistent differential calculus on the coloured 
quantum plane within the framework of noncommutative geometry. In addition, we have performed a contraction on the coloured $q$-plane to obtain the coloured $h$-plane

corresponding to the Jordanian quantum group $G L_{h}^{\lambda, \mu}(2)$. The results obtained are valid also for higher-dimensional and multiparametric coloured quantum planes.

Before closing, it is pertinent to comment on a few applications of this work. Firstly, the calculus developed here equips us with essential ingredients necessary to develop further the notion of coloured quantum spaces vis-a-vis coloured version of the $q$ deformed quantum mechanical phase space. Secondly, it would be useful to construct a differential calculus on the coloured $h$-plane which could possibly be obtained by contraction of the calculus on the coloured $q$-plane presented here, and to investigate the intermediate coloured $(q, h)$-planes. One could then build up a whole class of differential calculi on various different coloured quantum planes including the supersymmetric (graded) versions. The coloured Manin plane also forms interesting example of quantum homogeneous spaces [15], the geometric nature of which is currently being studied from several aspects.

Besides, it will be useful to investigate differential geometric structure on the underlying coloured Hopf algebras, which arose in the knot theoretic context. Also important to address are issues such as ring theoretic and quantum matrix theory aspects of coloured quantum groups. It would be of interest to explore further relation with the already well-known $q$-Heisenberg algebras, as well as the possibility of coloured generalisation of some new quantum groups [16] given recently.

\section{REFERENCES}

[1] Yu. I. Manin, Quantum groups and noncommutative geometry, Preprint Montreal University CRM-1561 (1988).

[2] J. Wess and B. Zumino, Covariant differential calculus on the quantum hyperplane, Nucl. Phys. (Proc. Suppl.) 18B (1990) $302-312$.

[3] P. Parashar, Multiparametric Deformations of Quantum Vector Spaces, Ph.D. Thesis, University of Delhi, 1992.

[4] A. Aghamohammadi, The two-parametric extension of $h$-deformation of $G L(2)$, and the differential calculus on its quantum plane, Modern Phys. Lett. A 8 (1993) 2607 - 2613.

[5] S. Cho, J. Madore and K. S. Park, Noncommutative geometry of the $h$-deformed quantum plane, J. Phys. A 31 (1998) 2639 - 2654. 
[6] J. Madore, An Introduction to Noncommutative Differential Geometry and its Physical Applications, Cambridge University Press, 1995.

[7] B. Basu-Mallick, Coloured extensions of $G L_{q}(2)$ quantum group and related noncommutative planes, Internat. J. Modern Phys. A 10, (1995) 2851 - 2864.

[8] C. Quesne, Coloured quantum universal enveloping algebras, J. Math. Phys. 38 (1997) 6018 - 6039; Duals of coloured quantum universal enveloping algebras and coloured universal Tmatrices, J. Math. Phys. 39 (1998) 1199 - 1222.

[9] P. Parashar, Jordanian $U_{h, s} g l(2)$ and its coloured realisation, Lett. Math. Phys. 45 (1998) 105 -112 .

[10] D. Parashar and R. J. McDermott, Contraction of the $G_{r, s}$ quantum group to its nonstandard analogue and corresponding coloured quantum groups, J. Math. Phys. 41 (2000) 2403 - 2416.

[11] P.-G. Luan, H. C. Lee and R. B. Zhang, Coloured solutions of the Yang-Baxter equation from representations of $U_{q} g l(2), J$. Math. Phys. 41 (2000) 6529 - 6543.

[12] D. Parashar, Duality for coloured quantum groups, Lett. Math. Phys. 53 (2000) 29 - 40.

[13] L. D. Faddeev, N. Y. Reshetikhin and L. A. Takhtajan, Quantisation of Lie groups and Lie algebras, Leningrad Math. J. 1 (1990) 193 - 225.

[14] A. Aghamohammadi, M. Khorrami and A. Shariati, Jordanian deformation of SL(2) as a contraction of its Drinfeld-Jimbo deformation, J. Phys. A 28 (1995) L225 - L331.

[15] F. Bonechi, N. Ciccoli, R. Giachetti, E. Sorace and M. Tarlini, The coisotropic subgroup structure of $S L_{q}(2, \mathbb{R})$, J. Geom. Phys. 37 (2001) 190 - 200, and references therein.

[16] S. Rodriguez-Romo and E. Taft, private communication (2001).

Max-Planck-Institute for Mathematics in the Sciences, Inselstrasse 22-26, D-04103 LeIPZIG, GERMANy

E-mail address: Deepak.Parashar@mis.mpg.de

$U R L:$ http://personal-homepages.mis.mpg.de/parashar/ 